\title{
A comparison between the 2010 and 2005 basic life support guidelines during simulated hypogravity and microgravity
}

Thais Russomano ${ }^{1,2^{*}}$, Justin H Baers ${ }^{1,2}$, Rochelle Velho ${ }^{1}$, Ricardo B Cardoso ${ }^{1}$, Alexandra Ashcroft ${ }^{1,2}$, Lucas Rehnberg ${ }^{1}$, Rodrigo D Gehrke ${ }^{1}$, Mariana K P Dias ${ }^{1}$ and Rafael R Baptista ${ }^{1}$

\begin{abstract}
Background: Current 2010 terrestrial $\left(1 \mathrm{G}_{z}\right)$ CPR guidelines have been advocated by space agencies for hypogravity and microgravity environments, but may not be feasible. The aims of this study were to (1) evaluate rescuer performance over 1.5 min of external chest compressions (ECCs) during simulated Martian hypogravity $\left(0.38 \mathrm{G}_{z}\right)$ and microgravity $(\mu \mathrm{G})$ in relation to $1 \mathrm{G}_{z}$ and rest baseline and (2) compare the physiological costs of conducting ECCs in accordance with the 2010 and 2005 CPR guidelines.

Methods: Thirty healthy male volunteers, ranging from 17 to 30 years, performed four sets of 30 ECCs for 1.5 min using the 2010 and 2005 ECC guidelines during $1 \mathrm{G}_{\mathrm{z}}, 0.38 \mathrm{G}$ and $\mu \mathrm{G}$ simulations (Evetts-Russomano (ER) method), achieved by the use of a body suspension device. ECC depth and rate, range of elbow flexion, post-ECC heart rate (HR), minute ventilation $\left(V_{E}\right)$, peak oxygen consumption $\left(\mathrm{VO}_{2}\right.$ peak) and rate of perceived exertion (RPE) were measured.

Results: All volunteers completed the study. Mean ECC rate was achieved for all gravitational conditions, but true depth during simulated microgravity was not sufficient for the $2005(28.5 \pm 7.0 \mathrm{~mm})$ and $2010(32.9 \pm 8.7 \mathrm{~mm})$ guidelines, even with a mean range of elbow flexion of $15^{\circ}$. $\mathrm{HR}, V_{E}$ and $\mathrm{VO}_{2}$ peak increased to an average of $136 \pm 22 \mathrm{bpm}, 37.5 \pm 10.3$ $\mathrm{L} \cdot \mathrm{min}^{-1}, 20.5 \pm 7.6 \mathrm{~mL} \cdot \mathrm{kg}^{-1} \cdot \mathrm{min}^{-1}$ for $0.38 \mathrm{G}$ and $161 \pm 19 \mathrm{bpm}, 58.1 \pm 15.0 \mathrm{~L} \cdot \mathrm{min}^{-1}, 24.1 \pm 5.6 \mathrm{~mL} \cdot \mathrm{kg}^{-1} \cdot \mathrm{min}^{-1}$ for $\mu \mathrm{G}$ from a baseline of $84 \pm 15 \mathrm{bpm}, 11.4 \pm 5.9 \mathrm{~L} \cdot \mathrm{min}^{-1}, 3.2 \pm 1.1 \mathrm{~mL} \cdot \mathrm{kg}^{-1} \cdot \mathrm{min}^{-1}$, respectively. RPE was the only variable to increase with the 2010 guidelines.

Conclusion: No additional physiological cost using the 2010 basic life support (BLS) guidelines was needed for healthy males performing ECCs for 1.5 min, independent of gravitational environment. This cost, however, increased for each condition tested when the two guidelines were compared. Effective ECCs were not achievable for both guidelines in simulated $\mu \mathrm{G}$ using the ER BLS method. This suggests that future implementation of an ER BLS in a simulated $\mu \mathrm{G}$ instruction programme as well as upper arm strength training is required to perform effective BLS in space.
\end{abstract}

Keywords: Basic life support, CPR guidelines, Hypogravity, Microgravity

\section{Background}

Human exploration of space is curtailed by the physiological and technical impact of reduced gravity. Nevertheless, it has provoked a fascination in mankind as limitless as the void of space itself. Aerospace medicine and physiology are evolving in tandem with explorer-class missions

\footnotetext{
* Correspondence: trussomano@hotmail.com

'Microgravity Centre, School of Engineering, PUCRS, Porto Alegre 90619-900, Brazil

${ }^{2}$ Centre of Human and Aerospace Physiological Sciences, School of Biomedical Sciences, KCL, London WC2R 2LS, UK
}

to accommodate the challenges associated with maintaining the safety, health and optimum performance of astronauts during spaceflights.

All organ systems are affected by exposure to extraterrestrial environments. Alterations to cardiovascular physiology with reduced gravity manifest acutely and chronically [1]. Reduced-gravity environments cause the cardiovascular system to undergo adaptive functional and structural changes. Microgravity induces a reduction in hydrostatic pressure, causing a cephalic redistribution of blood and body fluids. This headward shift is responsible

\section{Biomed Central}


for the 'puffy-face \& bird-leg' appearance of astronauts in space. The cardiovascular system adapts to microgravity by reducing blood volume by approximately $20 \%$, which is in part responsible for the orthostatic intolerance commonly found post-spaceflight. A reduction in heart size was also observed in microgravity [2]. However, based on data from space missions, it is suggested that such cardiovascular alterations do not lead to important cardiac dysfunction or dysrrhythmias. Therefore, the possibility of cardiac deconditioning developing into a life-threatening condition, such as a cardiac arrest, during short to moderate spaceflights is approximately $1 \%$ per year [3]. Nevertheless, with space agencies shifting their emphasis to lunar return missions and the eventual human exploration of Mars, the likelihood for cardiovascular issues to manifest themselves will be further enhanced with increasing space mission length.

An explorer-class mission to Mars will require approximately 2.4 years for completion: a 6-month flight to Mars, an approximate 500-day surface stay, and a 6-month return flight to Earth [4]. The cumulative and interactive effects of physiological problems from a long-term spaceflight could be potentially devastating for crewmembers. Prolonged exposure to reduced gravity may result in altered heart conduction and repolarisation, predisposing astronauts to cardiac dysrrhythmias [5]; electrical heart instability, in conjunction with encountered biodynamic stressors, presents the disturbing possibility of cardiac arrest in astronauts partaking in lengthy missions.

Further to exploration-class missions, the global private sector is having a greater influence on space ventures. The introduction of civilian tourist space travel broadens the population who may be subjected to the pertinent aspects of cardiovascular risks associated with spaceflight. Survey data show the demographics expected for suborbital spaceflight participants to be $70 \%$ male with an average of 57 years of age, $22 \%$ of which were older than 65 years [6]. This suggests that the expected population engaging in civilian spaceflight will be more likely to harbour subclinical cardiovascular conditions, hence increasing the probability of a cardiac event. Currently, international space institutions are refraining from imposing safety regulations, stating that there are no medical requirements for space tourism passengers and that only minimum training is required on how to respond to emergency situations [7].

Effective management of acute and chronic medical emergencies, such as basic life support (BLS), is vital on missions to ensure astronaut and tourist safety. External chest compressions (ECCs) constitute the core of BLS and must continue until advanced life support (ALS) can commence to maintain adequate perfusion to vital organs. The collaborative algorithm between the American Heart Association and the European Resuscitation Council for adult BLS delineates key steps required for effective terrestrial cardiopulmonary resuscitation (CPR) and was updated in 2010 [8]. These new guidelines place more emphasis on ECCs than ventilation. The previous airways-breathing-circulation 'A-B-C' algorithm has been altered to 'C-A-B'. This ensures rapid blood distribution to target areas whilst oxygen saturation is sufficiently high. It is now essential to perform ECCs of adequate depth (minimum $50 \mathrm{~mm}$ ) and rate (100 compressions $\cdot \mathrm{min}^{-1}$ ) [8].

Terrestrial $\left(1 G_{z}\right)$ CPR guidelines have been advocated by international space agencies for hypogravity and microgravity environments. Nonetheless, performing ECCs during spaceflight is more challenging due to reduced gravity [9]. Previous studies have shown the 2005 CPR guidelines to be feasible for simulated hypogravity and microgravity conditions. However, current guidelines, which require deeper ECCs, may not be feasible without compromising the rescuer's health and may go beyond the rescuer's physical capability; therefore, a comparison between the 2005 and 2010 CPR guidelines in hypogravity and microgravity environments is needed.

This investigation aimed to evaluate rescuer performance over $1.5 \mathrm{~min}$ of ECCs during simulated Martian hypogravity and microgravity in relation to $1 \mathrm{G}_{\mathrm{z}}$ and additionally compare the physiological costs of conducting ECCs in accordance with the 2005 and 2010 CPR guidelines. It was hypothesised that current ECC depth and frequency guidelines should be achievable for all simulated gravitational conditions. However, the 2010 ECC guidelines were expected to be more physiologically demanding in proportion to the reduction in simulated gravity.

\section{Methods}

\section{Study design}

The protocol included performing four sets of 30 ECCs over a period of $1.5 \mathrm{~min}$ in accordance to the 2005 and 2010 CPR guidelines during 1Gz, ground-based Martian hypogravity $\left(0.38 \mathrm{G}_{\mathrm{z}}\right)$ and microgravity $(\mu \mathrm{G})$ simulations at the John Ernsting Aerospace Physiology Laboratory, Microgravity Centre, Pontifícia Universidade Catolica do Rio Grande do Sul (PUCRS), Brazil. The study employed a within-volunteer repeated measures design, with each volunteer being their own control. The order of simulated gravitational conditions and CPR guidelines were randomised. The study protocol was approved by the Ethics and Research Committees of PUCRS.

\section{Volunteers}

A total of 30 healthy male volunteers, ranging from 17 to 30 years of age, served as rescuers performing CPR. They were recruited on a voluntary basis and signed a consent form prior to the beginning of the study. 


\section{Equipment and materials}

A standard CPR mannequin (Resusci Anne Skill Reporter, Laerdal Medical Ltd., Orpington, UK) was modified to include a linear displacement transducer capable of measuring ECC depth and rate. The mannequin's chest steel spring depressed $1 \mathrm{~mm}$ with every $1 \mathrm{~kg}$ of weight that was applied to it. Real-time feedback of each ECC was provided to the volunteers via a modified electronic guiding system with a light-emitting diode (LED) display. The LED display consisted of a series of coloured lights that indicated depth of ECCs (red, 0-39 mm; yellow, 40-49 mm; green, 50-60 $\mathrm{mm})$. An ECC rate of 100 compressions min $^{-1}$ was established using an electronic metronome. A 6-s interval between each ECC set represented the time taken for two mouth-to-mouth ventilations.

A custom-built body suspension device (BSD) was used to simulate reduced gravitational fields (developed by the Microgravity Centre, PUCRS). It is pyramidal in shape and consists of carbon steel bars of $6 \mathrm{~cm} \times 3 \mathrm{~cm}$ thickness (base area, $300 \mathrm{~cm} \times 226 \mathrm{~cm}$; height, $200 \mathrm{~cm}$ ). It comprises of a body harness and counterweight system made of 20 bars of $5 \mathrm{~kg}$ each (Figure 1).

For simulated $0.38 G_{z}$, the steel cable connected the counterweights through a pulley system to the harness worn by the volunteer. The necessary counterweights were calculated using Equations 1 and 2 [10]:

$$
\begin{aligned}
& R M=\frac{0.6 \mathrm{BM} \times \mathrm{SGF}}{1 G} \\
& C W=0.6 \mathrm{BM}-\mathrm{RM}
\end{aligned}
$$

where RM is the relative mass (in $\mathrm{kg}$ ), $0.6 \mathrm{BM}$ is the percentage of total body mass, SGF is the simulated gravitational force $\left(\mathrm{m} \cdot \mathrm{s}^{-2}\right), 1 \mathrm{G}=9.81 \mathrm{~m} \cdot \mathrm{s}^{-2}$ and $\mathrm{CW}$ is the counterweight (in $\mathrm{kg}$ ).

During the performance of ECCs, the mannequin was placed supine on the floor with the volunteer adopting the terrestrial CPR position.

For simulated $\mu \mathrm{G}$, volunteers were suspended by the body harness via the use of the steel cross bar (1205.0 $\mathrm{mm} \times 27.5 \mathrm{~mm}$ ). A static nylon rope was attached to the steel wiring of the cross bar, with carabineers fastened at each end. These were clipped to corresponding hip attachments of the body harness. A safety carabineer was also attached to the volunteer's back.

The mannequin was fully suspended to allow the performance of the Evetts-Russomano (ER) BLS technique. In order to perform the ER technique, the volunteer places his left leg over the mannequin's right shoulder and his right leg around the torso and across the back of the mannequin. The left and right ankles cross in the inter-scapula area of the mannequin for added stability. The application of force to the chest of the mannequin will then be countered by the volunteer's legs and feet and is achieved by the flexion and extension of the volunteer's arms [11].

Angle of elbow flexion was measured using a custombuilt electrogoniometer on the volunteer's dominant arm (developed by the Microgravity Centre, PUCRS). The electrogoniometer consisted of two aluminium bars (200.0 $\mathrm{mm} \times 20.0 \mathrm{~mm} \times 3.0 \mathrm{~mm}$ ) covered with rubber material and was fastened over the volunteer's lateral epicondyle via a series of straps; this allowed the change in flexion/extension (from $0^{\circ}$ to $90^{\circ}$ ) to be accurately measured. The device was connected with a linear $10 \mathrm{k} \Omega$ potentiometer and powered by a $5-\mathrm{V}$ power source.

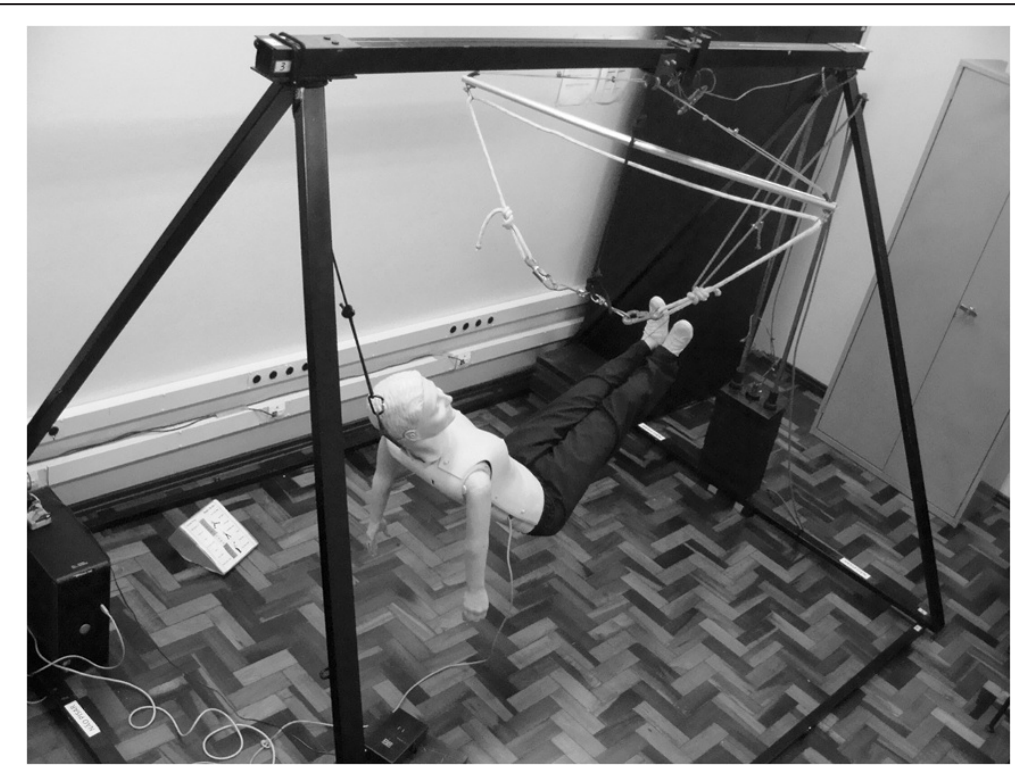

Figure 1 Body suspension device with mannequin fully suspended simulated microgravity. 
An Aerosport VO2000 analyser (MedGraphics, Saint Paul, MN, USA) recorded minute ventilation $\left(V_{\mathrm{E}}\right)$ and oxygen consumption per minute $\left(\mathrm{VO}_{2}\right) . \mathrm{VO}_{2}$ was standardised, calculated and recorded directly by the computerized ergospirometric system used (Aerograph 4.3, AeroSport Inc., Ann Arbor, MI, USA).

An Onyx 9500 fingertip pulse oximeter measured heart rate (HR; Nonin Medical Inc., Plymouth, MN, USA). The Borg scale measured rate of perceived exertion [12].

\section{Protocol}

Anthropometric characteristics (height in $\mathrm{m}$, weight in $\mathrm{kg}$ ) were measured, and body mass index (BMI; $\mathrm{kg} \cdot \mathrm{m}^{-2}$ ) was calculated from them. Volunteers were first familiarised with the equipment, as well as both terrestrial CPR and ER techniques; volunteers were required to demonstrate that they had mastered both BLS methods.

Volunteers rested for $5 \mathrm{~min}$ prior to BLS to record baseline values. They then performed four sets of ECCs over a period of $1.5 \mathrm{~min}$ in accordance with the 2005 and 2010 ECC guidelines at $1 G_{z}$ followed by the two gravitational simulations. A minimum of $10 \mathrm{~min}$ rest was given to volunteers between each set of ECCs.

ECC frequency and depth, as well as angle of elbow flexion, were measured throughout the experiment. Exhaled gases were sampled continuously and analysed every three breaths. Heart rate was recorded before (resting heart rate) and immediately after the completion of each protocol. After four sets of ECCs, subjective appraisal of exertion using the Borg scale was noted.

The Aerosport VO2000 analyser used its own software and was auto-calibrated prior to each protocol. The mannequin's chest system was calibrated between volunteers using inputs of 0 and $60 \mathrm{~mm}$. The elbow electrogoniometer was calibrated prior to each protocol using two points: full extension of the arm $\left(0^{\circ}\right)$ and measured $90^{\circ}$ flexion.

A DataQ acquisition device with eight analogue and six digital channels, 10 bits of measurement accuracy, rates up to 14,400 samples $\cdot \mathrm{s}^{-1}$ and USB interface was used (DATA$\mathrm{Q}$ Instruments Inc., Akron, OH, USA). The device supported a full-scale range of $\pm 10 \mathrm{~V}$ and a resolution of \pm 19.5 $\mathrm{mV}$. WinDaq data acquisition software allowed for the conversion of volts to the necessary units used. Two input channels were used during data collection: one from the chest system of the mannequin and the other from the elbow electrogoniometer.

\section{Data analysis}

Data of physiological variables, which were determined by either averaging the last $30 \mathrm{~s}$ of exercise or comparing the last $30 \mathrm{~s}$ of exercise to baseline state and ECC depth, rate and elbow flexion, were reported as mean values $( \pm \mathrm{SD})$. Percentage of maximum HR was calculated by comparing post-ECC HR with theoretical maximum HR (calculated using the 220-age equation) [13]. $\mathrm{VO}_{2}$ peak represents the highest recorded $\mathrm{VO}_{2}$ during the four ECC sets. Elbow flexion was calculated as a range from the minimum to maximum angle of an individual ECC. The ECC depth was analysed in two different ways: maximum depth $\left(D_{\text {Max }}\right)$ achieved and true depth $\left(D_{\mathrm{T}}\right)$, which was calculated using Equation 3:

$$
D_{T}=D_{\mathrm{Max}}-D_{\text {IRecoil }}
$$

where $D_{\mathrm{T}}$ is the true depth of external chest compression, $D_{\mathrm{Max}}$ is the maximum depth of external chest compression and $D_{\text {IRecoil }}$ is the depth of inadequate recoil, which is the distance not decompressed between subsequent external compressions.

The measures were derived post hoc from the data files using GraphPad Prism v5.0a for analysis. Statistical comparisons were performed on physiological variables using a one-way, non-parametric ANOVA test and on ECCs and elbow flexion data using a two-way ANOVA. A 95\% confidence interval calculation around the mean was used. The level of significance was set $a$ priori as $p \leq 0.05$.

\section{Results}

All 30 volunteers completed the protocol. Mean $( \pm$ SD) age, weight, height and BMI were $22.5( \pm 3.5)$ years, 78.2 $( \pm 13.1) \mathrm{kg}, 1.80( \pm 0.07) \mathrm{m}$ and $23.3( \pm 2.9) \mathrm{kg} \cdot \mathrm{m}^{-2}$, respectively.

The mean $\left( \pm\right.$ SD) $D_{\text {Max }}$ of all four sets for $1 G_{z}$ and the simulated gravitational environments for the 2005 and 2010 ECC guidelines is presented in Figure 2A,B. All volunteers were able to abide by the 2005 and 2010 ECC guidelines at $1 \mathrm{G}_{\mathrm{z}}(47.1( \pm 3.0)$ and $57.0( \pm 2.3) \mathrm{mm})$ and simulated $0.38 \mathrm{G}_{\mathrm{z}}(46.2( \pm 3.6)$ and $55.1( \pm 3.7) \mathrm{mm})$. For simulated $\mu \mathrm{G}$, the mean ECC $D_{\text {Max }}$ obtained using the ER method fell $0.2 \mathrm{~mm}$ below the 2005 guidelines (39.8 $( \pm 8.3) \mathrm{mm})$, and there was considerable variation in the range of ECC $D_{\text {Max }}$. Eleven volunteers were able to adhere to the 2010 ECC guidelines in simulated $\mu \mathrm{G}$, and the mean $D_{\text {Max }}$ fell short of the 50 -mm effective limit $(44.9( \pm 10.9) \mathrm{mm})$. However, not all volunteers allowed full recoil of the mannequin's chest for the three gravitational conditions. The mean $( \pm \mathrm{SD}) D_{\text {IRecoil }}$ for $1 G_{\mathrm{z}}$, $0.38 \mathrm{G}_{\mathrm{z}}$ and $\mu \mathrm{G}$ were $6.7( \pm 4.9), 2.5( \pm 2.2)$ and 11.5 $( \pm 5.5) \mathrm{mm}$ for $2005 \mathrm{ECC}$ guidelines and $4.6( \pm 3.5), 1.6$ $( \pm 1.8)$ and $11.9( \pm 5.7) \mathrm{mm}$ for the 2010 ECC guidelines, respectively. For both ECC guidelines, $D_{\text {IRecoil was less }}$ during the Martian simulation and higher during simulated $\mu \mathrm{G}$.

The mean $( \pm \mathrm{SD}) D_{\mathrm{T}}$ of the individual ECC sets per condition, calculated from $D_{\text {IRecoil }}$ to $D_{\text {Max }}$, is depicted in Figure 3A,B and Table 1 . Mean $D_{\mathrm{T}}$ was within the effective limits set by the 2005 and 2010 ECC guidelines 
in the $1 G_{\mathrm{z}}$ control environment for all four ECC sets. For the 2005 ECC guidelines, the mean $D_{\mathrm{T}}$ for the last three ECC sets was above the effective lower limit for simulated $0.38 \mathrm{G}_{\mathrm{z}}$ compared to $1 \mathrm{G}_{\mathrm{z}}$. In contrast, the mean $D_{\mathrm{T}}$ of ECCs was below the effective lower limit in simulated $\mu \mathrm{G}$ compared to the $1 G_{z}$ control environment for both ECC guidelines and for all four ECC sets.

The mean $( \pm \mathrm{SD})$ ECC rate was successfully maintained above 100 compressions. $\mathrm{min}^{-1}$ for each set within each gravitational condition, with reference to both ECC guidelines (Table 1).

The mean $( \pm S D)$ ranges of elbow flexion of the volunteer's dominant arm at $1 \mathrm{G}_{\mathrm{z}}$ during the 2005 and 2010 ECC guidelines were $3.4^{\circ}\left( \pm 2.0^{\circ}\right)$ and $4.3^{\circ}\left( \pm 2.8^{\circ}\right)$, respectively. The range of elbow flexion increased to $10.6^{\circ}$ $\left( \pm 6.8^{\circ}\right)$ during the 2005 ECC guidelines and to $14.0^{\circ}$ $\left( \pm 8.1^{\circ}\right)$ during the 2010 ECC guidelines in simulated $0.38 \mathrm{G}_{\mathrm{z}}$. When using the ER method in simulated $\mu \mathrm{G}$, ranges of elbow flexion of the volunteer's dominant arm during the 2005 and 2010 ECC guidelines were $15.5^{\circ}$ $( \pm 8.7)^{\circ}$ and $16.5^{\circ}( \pm 10.1)^{\circ}$. No difference in range of elbow flexion was observed between ECC guidelines for either simulated reduced gravity conditions (Figure 4).

The mean $( \pm S D)$ rescuer $\mathrm{HR}$ at baseline, post-ECC, as well as percent change and percentage of maximum $\mathrm{HR}$ in all three gravitational conditions is illustrated in Table 2. There was an increment in HR responses postECC for simulated $0.38 G_{z}$ and $\mu G$. No differences between ECC guidelines were noted.

Mean $( \pm \mathrm{SD})$ rescuer $V_{\mathrm{E}}$ for $1 \mathrm{G}_{\mathrm{z}}, 0.38 \mathrm{G}_{\mathrm{z}}$ and $\mu \mathrm{G}$ increased from $11.4( \pm 5.9) \mathrm{L} \cdot \mathrm{min}^{-1}$ at rest to $23.8( \pm 6.2)$, $34.4( \pm 10.4)$ and $55.1( \pm 15.6) \mathrm{L} \cdot \mathrm{min}^{-1}$ for the $2005 \mathrm{ECC}$ guidelines and $27.5( \pm 7.9), 40.6( \pm 10.2)$ and $61.1( \pm 14.4)$ $\mathrm{L} \cdot \mathrm{min}^{-1}$ for the 2010 ECC guidelines, respectively. With respect to both ECC guidelines, there was no significant difference in the increase in $V_{\mathrm{E}}$ from rest for the three gravitational conditions (Figure 5A). During the last $30 \mathrm{~s}$ of ECCs, $V_{\mathrm{E}}$ increased by $153.0 \%, 275.8 \%$ and $490.1 \%$ at $1 G_{z}, 0.38 G_{z}$ and $\mu G$ for the 2005 ECC guidelines. An increase of $194.8 \%, 334.9 \%$ and $568.1 \%$ was seen for the 2010 ECC guidelines, respectively.

During the performance of ECCs, the mean $( \pm \mathrm{SD})$ rescuer $\mathrm{VO}_{2}$ increased from $3.2( \pm 1.1) \mathrm{mL} \cdot \mathrm{kg}^{-1} \cdot \mathrm{min}^{-1}$ at rest to peak levels of $14.8( \pm 5.0) \mathrm{mL} \cdot \mathrm{kg}^{-1} \cdot \mathrm{min}^{-1}$ at $1 \mathrm{G}_{\mathrm{z}}$, $19.3( \pm 7.1) \mathrm{mL} \cdot \mathrm{kg}^{-1} \cdot \mathrm{min}^{-1}$ at $0.38 \mathrm{G}_{\mathrm{z}}$ and $23.5( \pm 5.1)$ $\mathrm{mL} \cdot \mathrm{kg}^{-1} \cdot \mathrm{min}^{-1}$ at $\mu \mathrm{G}$ for the $2005 \mathrm{ECC}$ guidelines. For the 2010 ECC guidelines, the increase was to $16.4( \pm 4.5)$ $\mathrm{mL} \cdot \mathrm{kg}^{-1} \cdot \mathrm{min}^{-1}$ at $1 \mathrm{G}_{\mathrm{z}}, 21.8( \pm 8.1) \mathrm{mL} \cdot \mathrm{kg}^{-1} \cdot \mathrm{min}^{-1}$ at $0.38 \mathrm{G}_{\mathrm{z}}$ and $24.7( \pm 6.2) \mathrm{mL} \cdot \mathrm{kg}^{-1} \cdot \mathrm{min}^{-1}$ at $\mu \mathrm{G}$ (Figure $5 \mathrm{~B}$ ). During the last $30 \mathrm{~s}$ of ECCs, $\mathrm{VO}_{2}$ increased by $283.3 \%$, $428.6 \%$ and $559.7 \%$ at $1 G_{z}, 0.38 G_{z}$ and $\mu G$ for the 2005 ECC guidelines. An increase of $367.7 \%, 509.0 \%$ and $590.3 \%$ was seen for the 2010 ECC guidelines, respectively. No difference was noted between ECC guidelines for all three gravitational conditions.

The Borg scale showed there was an increase in the mean $( \pm S D)$ rate of perceived exertion intra- and interconditions (Figure 6).

\section{Discussion}

Preparation for adverse cardiac events is vital to ensure the safety of space explorers, thus potentiating the development of the most effective protocol for BLS in simulated $0.38 \mathrm{G}_{\mathrm{z}}$ and $\mu \mathrm{G}$.

This study was the first of its kind to investigate the administration of effective ECCs using the 2010 ECC guidelines in comparison to the previous 2005 ECC guidelines during simulated $0.38 \mathrm{G}_{\mathrm{z}}$ and $\mu \mathrm{G}$, while looking at the physiological impact on the rescuer.

Both ECC guidelines emphasise that effective ECCs have two key components-adequate compression depth and rate-to ensure sufficient haemodynamics from time of arrest to application of ALS. When assessing the $D_{\text {Max }}$ achieved during ECCs, results from the $1 \mathrm{G}_{\mathrm{z}}$ and simulated $0.38 \mathrm{G}_{\mathrm{z}}$ sessions showed that all volunteers were able to perform according to both the 2005 and 2010 ECC standards. In fact, the ability of volunteers to abide by the previous $2005 \mathrm{ECC}$ guidelines at $1 \mathrm{G}_{\mathrm{z}}$ and during simulated
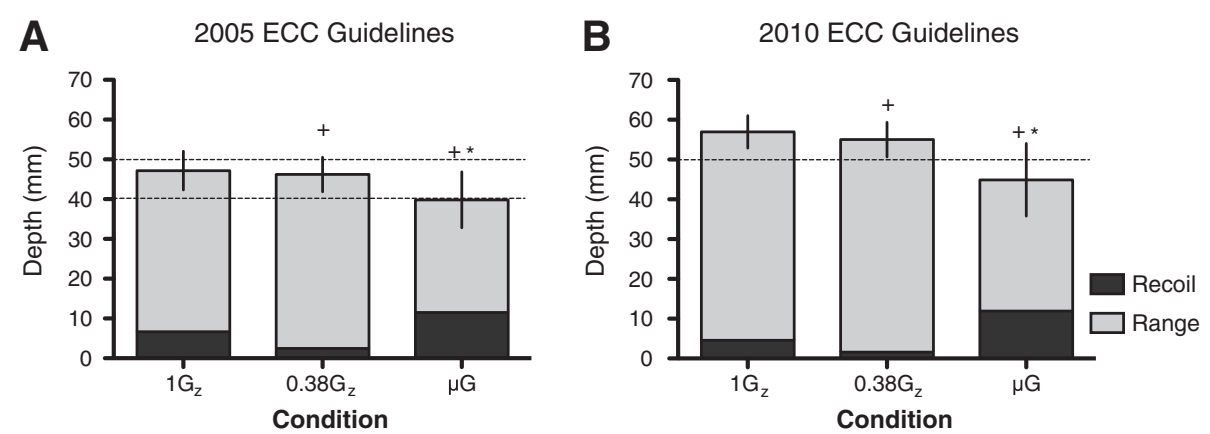

Figure 2 Mean $( \pm S D)$ maximum depth with depth of compressed chest post-inadequate recoil at $1 G_{z}, 0.38 G_{z}$ and $\mu G$. $(A)$ The 2005 ECC guidelines. (B) The 2010 ECC guidelines. The dashed line(s) depicts the effective limit(s) of depth for each respective guideline. $n=30 ;$ asterisk denotes significant difference in maximum depth to $1 \mathrm{G}_{z}$ control, $p<0.05$. The plus sign denotes significant difference in recoil to $1 \mathrm{G}_{z}$ control, $p<0.05$. 

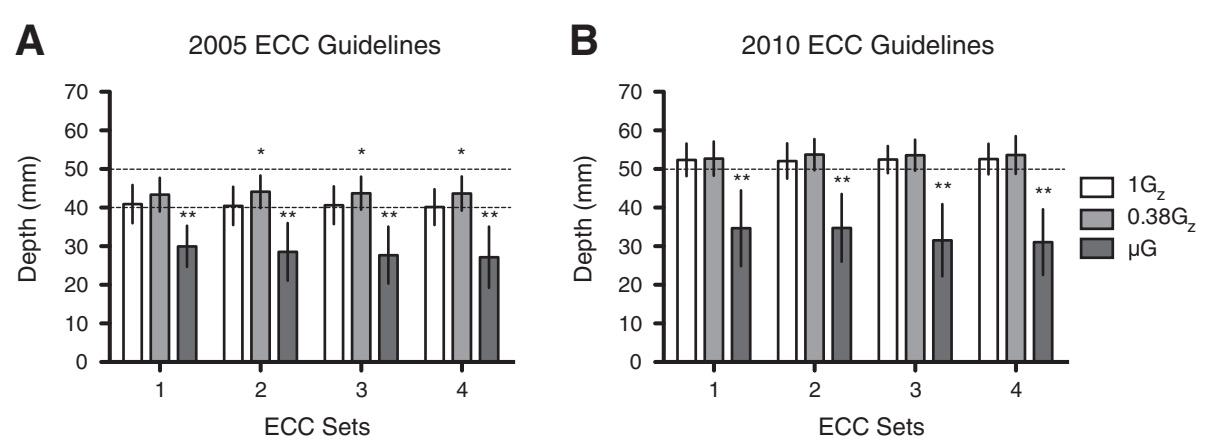

Figure 3 Mean $( \pm \mathrm{SD})$ true depth $\left(\boldsymbol{D}_{\mathbf{T}}\right)$ of $\mathrm{ECC}$ at $\mathbf{1} \mathrm{G}_{\mathbf{z}} \mathbf{\mathbf { 0 . 3 8 }} \mathbf{\mathbf { 3 }} \mathbf{z}$ and $\boldsymbol{\mu G}$. $(\mathbf{A})$ The $2005 \mathrm{ECC}$ guidelines. $(\mathbf{B})$ The $2010 \mathrm{ECC}$ guidelines. The dashed line (s) depicts the effective limit(s) of depth for each respective guideline. $n=30$; single asterisk denotes $p<0.05$, while double asterisk denotes $p<0.001$.

$0.38 \mathrm{G}_{\mathrm{z}}$ is in agreement with previous studies $[10,14]$. Although mean $D_{\text {Max }}$ did not meet the 2005 ECC guidelines during simulated $\mu \mathrm{G}$, a negligible difference of $0.2 \mathrm{~mm}$ would probably be deemed effective during in vivo BLS. However, the considerable inter-volunteer variability observed questions the efficacy of the ER method, which concurred with the findings of Rehnberg et al. [14]. Mean $D_{\mathrm{Max}}$ failed to abide by the 2010 ECC guidelines, which corresponds with the findings of Kordi et al. [15] (Figure 2B).

Previous studies noted that volunteers were failing to consistently allow full chest recoil using the ER method during simulated $\mu \mathrm{G}$ (Figure 2) [14]. This can be detrimental to the effectiveness of BLS, as incomplete decompression decreases the change in thoracic pressure and thereby reduces perfusion to vital organs. To address this issue and a first for space CPR studies, ECC $D_{\mathrm{T}}$ was calculated by adjusting for $D_{\text {IRecoil }}$ (Figure 3 and Table 1).

When assessing ECC $D_{\mathrm{T}}$, all 30 volunteers failed to abide by both ECC guidelines during simulated $\mu \mathrm{G}$ using the ER method. This could be attributable to rescuers inadequately decompressing between individual ECCs or interruptions during ECCs when using the ER position in simulated $\mu \mathrm{G}$.

The inadequate decompressions between ECCs may be due to rescuers focussing on achieving the 100 compressions $\cdot \mathrm{min}^{-1}$ rate set by guidelines during simulated $\mu \mathrm{G}$. This is supported by mean ECC rate in keeping with both sets of ECC guidelines, whilst true depth of individual ECC sets was not (Table 1). This is not in accordance with a previous parabolic flight study using the ER method that found ECC rate to be lower whilst ECC depth remained adequate for the used ECC guidelines at that time, which were the same as 2005 . These findings, however, may represent a limitation of the BSD system. The parabolic study had a sample size of 3 and was able to adhere to ECC guidelines even with such a small window of freefall, approximately $20 \mathrm{~s}$ per parabola [11].

In addition, the high SD seen in Table 1, which represents the inter-volunteer variability for ECC rate,

Table 1 Mean $( \pm S D)$ true depth $\left(D_{T}\right)$ and rate of individual $E C C$ sets at $1 G_{z}, 0.38 G_{z}$ and $\mu \mathrm{G}$

\begin{tabular}{|c|c|c|c|c|c|c|}
\hline \multirow{2}{*}{$\begin{array}{l}\text { Gravitational } \\
\text { condition }\end{array}$} & \multirow{2}{*}{$\begin{array}{c}\text { ECC } \\
\text { guidelines }\end{array}$} & \multirow[t]{2}{*}{ ECC } & \multicolumn{4}{|c|}{ ECC sets } \\
\hline & & & 1 & 2 & 3 & 4 \\
\hline \multirow[t]{4}{*}{$1 \mathrm{G}_{\mathrm{z}}$} & 2005 & Depth (mm) & $40.9( \pm 5.0)$ & $40.4( \pm 5.0)$ & $40.6( \pm 4.9)$ & $40.1( \pm 4.6)$ \\
\hline & & Rate (comp $\min ^{-1}$ ) & $104( \pm 5)$ & $105( \pm 5)$ & $105( \pm 6)$ & $105( \pm 5)$ \\
\hline & 2010 & Depth (mm) & $52.4( \pm 4.2)$ & $52.1( \pm 4.6)$ & $52.5( \pm 3.5)$ & $52.6( \pm 3.9)$ \\
\hline & & Rate (comp.min ${ }^{-1}$ ) & $105( \pm 4)$ & $104( \pm 4)$ & $104( \pm 3)$ & $104( \pm 3)$ \\
\hline \multirow[t]{4}{*}{$0.38 G_{z}$} & 2005 & Depth (mm) & $43.4( \pm 4.4)$ & $44.1( \pm 4.2)^{*}$ & $43.7( \pm 4.3)^{*}$ & $43.7( \pm 4.4)^{*}$ \\
\hline & & Rate (comp.min ${ }^{-1}$ ) & $103( \pm 6)$ & $104( \pm 6)$ & $104( \pm 5)$ & $103( \pm 5)$ \\
\hline & 2010 & Depth (mm) & $52.7( \pm 4.4)$ & $53.7( \pm 4.0)$ & $53.6( \pm 4.0)$ & $53.6( \pm 4.9)$ \\
\hline & & Rate (comp.min ${ }^{-1}$ ) & $103( \pm 6)$ & $103( \pm 5)$ & $103( \pm 5)$ & $103( \pm 5)$ \\
\hline \multirow[t]{4}{*}{$\mu G$} & 2005 & Depth (mm) & $30.0( \pm 5.3)^{* *}$ & $28.5( \pm 7.5)^{* *}$ & $27.7( \pm 7.4)^{* *}$ & $27.1( \pm 7.9)^{* *}$ \\
\hline & & Rate (comp. $\mathrm{min}^{-1}$ ) & $105( \pm 7)$ & $106( \pm 5)$ & $105( \pm 5)$ & $106( \pm 5)$ \\
\hline & 2010 & Depth (mm) & $34.7( \pm 9.8)^{* *}$ & $34.8( \pm 8.7)^{* *}$ & $31.5( \pm 9.4)^{* *}$ & $31.1( \pm 8.5)^{* *}$ \\
\hline & & Rate (comp.min ${ }^{-1}$ ) & $104( \pm 7)$ & $105( \pm 5)$ & $106( \pm 8)$ & $103( \pm 10)$ \\
\hline
\end{tabular}

$n=30 ; *^{*} p<0.05$ and ${ }^{* *} p<0.01$, significant difference from $1 \mathrm{G}_{\mathrm{z}}$ control. 


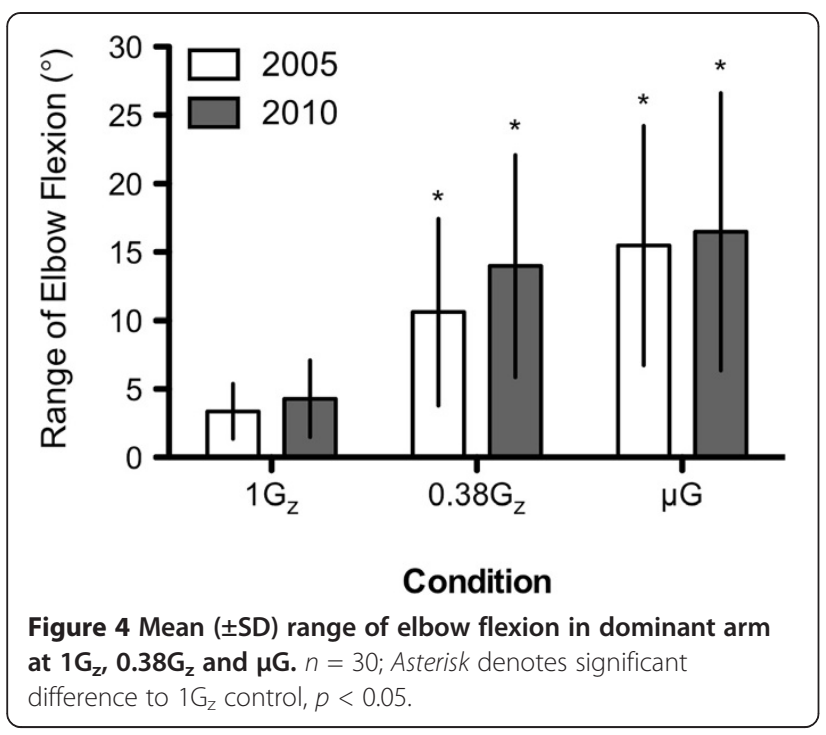

increases with time in simulated $\mu \mathrm{G}$. This suggests degradation of ECC rate during the course of BLS.

Overall, the results of this investigation suggest that the ECCs administered were ineffective in simulated $\mu \mathrm{G}$, as only mean ECC rate was adhered to, which would reduce the benefit to the casualty due to inadequate vital organ perfusion. This also indicates that the efficacy of the ER method is deficient at producing true depth of ECCs in a simulated $\mu \mathrm{G}$ environment, which contradicts previous studies only analysing maximum depth of ECCs and that did not account for $D_{\text {IRecoil }}$ of the mannequin [14].

The efficacy of ECCs is dependent on the physiological impact of $\mathrm{CPR}$ on the rescuer. Increased $\mathrm{HR}, V_{\mathrm{E}}$ and $\mathrm{VO}_{2}$ peak were inversely correlated with the simulated gravitational conditions studied, which indicates greater physical effort during the performance of BLS (Table 2 and Figure 5). The HR results support those found by both Dalmarco et al. [10] and Rehnberg et al. [14]. Furthermore, there was no difference in $\mathrm{HR}, V_{\mathrm{E}}$ and $\mathrm{VO}_{2}$ peak between ECC guidelines, which may imply that current ECC guidelines do not impose additional physical effort, unlike the simulated gravitational environment.
In our study, it is important to note that $\mathrm{VO}_{2}$ peak was measured and used as an estimation of $\mathrm{VO}_{2} \max$, allowing comparisons with previous literature findings to be drawn [16].

There are limited studies that evaluate $\mathrm{VO}_{2}$ max during or post-spaceflight, all of which are short-duration missions (<14 days) [17]. It has been hypothesised that appropriate exercise countermeasures may maintain $\mathrm{VO}_{2} \mathrm{max}$ during long-duration explorer-class missions. Thus, the additive effects of cardiac deconditioning would have less influence on the rescuer's aerobic capabilities to perform ECCs in $\mu \mathrm{G}$, making the physical difficulty of the ER method the key variable in performing effective ECCs.

Interestingly, decreases in $\mathrm{VO}_{2}$ max arise following reentry. Levine et al. [18] noted that after the SLS-1 and SLS-2 missions, six astronauts showed $\mathrm{VO}_{2}$ max levels of 2.1-2.9 $\mathrm{L} \cdot \mathrm{min}^{-1}$. In addition, the extra-vehicular activity (EVA) suit required for planetary surface exploration may also determine the level of cardiovascular exercise capacity. Studies at NASA's Johnson Space Center in simulated $0.38 \mathrm{G}_{\mathrm{z}}$ showed an increase in $\mathrm{VO}_{2}$ by an additional $20 \mathrm{~mL} \cdot \mathrm{kg}^{-1} \cdot \mathrm{min}^{-1}$ ( $40 \%$ of the volunteer's $\mathrm{VO}_{2} \max$ ) while wearing a Mark III prototype exploration EVA suit [19].

After a Martian landing, crewmembers will most likely be required to begin work immediately without a sufficient period for acclimatisation to $0.38 \mathrm{G}_{\mathrm{z}}$ [20]. This reduced aerobic capacity, in conjunction with orthostatic intolerance and impaired blood flow from long-term microgravity exposure, may significantly impact a crewmember's capability during emergencies or while assisting an incapacitated crewmate.

Although any attempt to administer CPR in an EVA suit is unlikely to be achievable, the physiological aspect would be interesting to consider. Therefore, the mean $\mathrm{VO}_{2}$ peak of a rescuer in an EVA suit would be 41.8 $\mathrm{mL} \cdot \mathrm{kg}^{-1} \cdot \mathrm{min}^{-1}$ in simulated $0.38 \mathrm{G}_{\mathrm{z}}$, which accounts for our $21.8 \mathrm{~mL} \cdot \mathrm{kg}^{-1} \cdot \mathrm{min}^{-1}$ (Figure $5 \mathrm{~B}$ ) and the expected additional $20 \mathrm{~mL} \cdot \mathrm{kg}^{-1} \cdot \mathrm{min}^{-1}$ from wearing an EVA suit [19]. For the average male weight $(78.2 \mathrm{~kg})$ in our study, this would equate to $3.3 \mathrm{~L} \cdot \mathrm{min}^{-1}$ after four sets of ECCs. This exceeds the $\mathrm{VO}_{2} \max$ of $2.9 \mathrm{~L} \cdot \mathrm{min}^{-1}$ found by

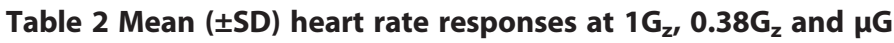

\begin{tabular}{|c|c|c|c|c|c|}
\hline \multicolumn{5}{|c|}{ Mean $( \pm S D)$, bpm } & \multirow{2}{*}{$\begin{array}{c}\text { ECC } \\
\text { guideline }\end{array}$} \\
\hline Baseline & Heart rate & $1 \mathrm{G}_{\mathrm{z}}$ & $0.38 G_{z}$ & $\mu G$ & \\
\hline \multirow[t]{6}{*}{$84( \pm 15)$} & HR post-ECC & $111( \pm 19)$ & $132( \pm 23)^{*}$ & $159( \pm 19)^{*}$ & 2005 \\
\hline & $\% \Delta$ & $33.8( \pm 18.4)$ & $60.0( \pm 25.8)^{*}$ & $94.7( \pm 33.9)^{*}$ & \\
\hline & $\% \operatorname{Max}$ & $56.1( \pm 9.4)$ & $66.9( \pm 11.6)^{*}$ & $80.7( \pm 9.9)^{*}$ & \\
\hline & HR post-ECC & $117( \pm 21)$ & $140( \pm 21)^{*}$ & $163( \pm 18)^{*}$ & 2010 \\
\hline & $\% \Delta$ & $41.4( \pm 22.8)$ & $71.2( \pm 30.5)^{*}$ & $98.8( \pm 35.4)^{*}$ & \\
\hline & $\% \operatorname{Max}$ & $59.2( \pm 10.9)$ & $71.1( \pm 10.7)^{*}$ & $82.3( \pm 9.4)^{*}$ & \\
\hline
\end{tabular}

$\mathrm{HR}$ responses are depicted as baseline and post-ECC values (bpm), percent change from baseline and percentage of maximum heart rate (maximum heart rate was calculated using the 220 -age equation). $n=30 ;{ }^{*} p<0.05$, significant difference from $1 \mathrm{G}_{\mathrm{z}}$ control. 


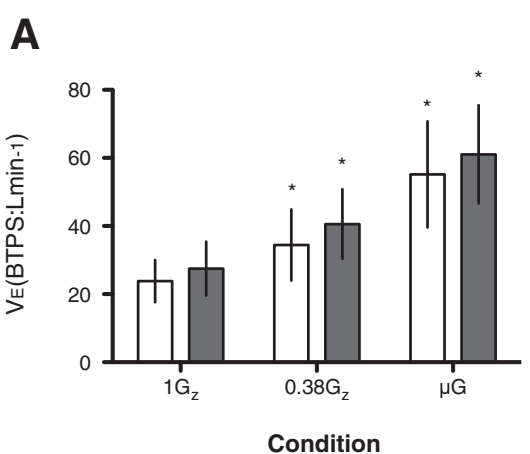

\section{B}

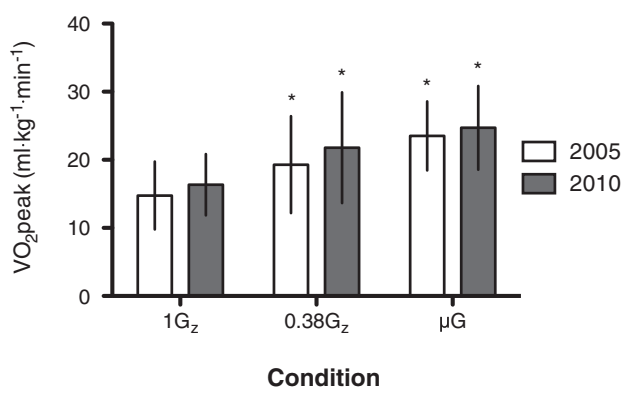

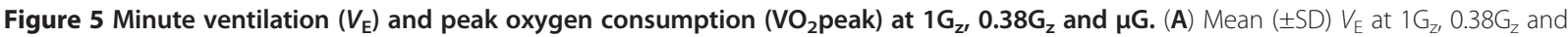
$\mu \mathrm{G}$. Baseline was $11.4( \pm 5.9) \mathrm{L} \cdot \mathrm{min}^{-1}$. (B) Mean $( \pm \mathrm{SD}) \mathrm{VO}_{2}$ peak normalised to weight at $1 \mathrm{G}_{\mathrm{z}}, 0.38 \mathrm{G}_{\mathrm{z}}$ and $\mu \mathrm{G}$. Baseline was $3.2( \pm 1.1) \mathrm{mL} \cdot \mathrm{kg}^{-1} \cdot \mathrm{min}^{-1}$. $n=30$; Asterisk denotes significant difference to $1 \mathrm{G}_{z}$ control, $p<0.05$.

Levine et al. [18]. This value may be an underestimation, as the casualty would also be wearing an EVA suit and the pressurisation of their suit would have to be overcome as well.

Furthermore, this study looked at the range of elbow flexion, while previous studies only took maximum elbow flexion into account $[10,14]$. The greater range of elbow flexion seen during simulated $0.38 \mathrm{G}_{\mathrm{z}}$ could be accredited to the recruitment of upper arm muscle groups to compensate for the reduction in upper body weight. The lack of difference in the range of elbow flexion for either ECC guidelines may indicate that the upper limb muscle groups are recruited in the same manner (Figure 4).

An increase in elbow flexion range was also noted between simulated $\mu \mathrm{G}$ and $1 \mathrm{G}_{\mathrm{z}}$. Using the $2005 \mathrm{ECC}$ guidelines during simulated $\mu \mathrm{G}$, the mean increase of $15.5^{\circ}$ $\left( \pm 8.7^{\circ}\right)$ in the volunteer's dominant arm was similar to the approximate $11^{\circ}\left( \pm 8.3^{\circ}\right)$ and $15^{\circ}\left( \pm 9.1^{\circ}\right)$ of the right and left arms, respectively, found by Rehnberg et al. [14]. However, it is important to highlight that the change from the terrestrial to the ER BLS position might have contributed to the recruitment of different muscle groups. Like simulated $0.38 \mathrm{G}_{\mathrm{z}}$, the lack of difference in the range of elbow flexion using the ER method for both ECC guidelines may indicate that the upper limb muscle groups are recruited in the same manner. This further suggests that guidelines are equally difficult in simulated $\mu \mathrm{G}$, as this correlates with the inability to achieve effective true ECC depth and the non-significant difference in physiological variables between guidelines (Figure 4).

Although the physiological variables measured were not different between guidelines, volunteers perceived current ECC guidelines to be more difficult (Figure 6). This might have been influenced by the fact that volunteers had a preconception that illuminating more LEDs for current ECC guidelines could have been less attainable.

This study is not without limitations, since it is based on the evaluation of healthy young males performing 1.5 min of BLS. The simulated gravitational environment, using a BSD, may not replicate all physiological effects secondary to reduced gravity exposure, apart from weight reduction, which is essential for successful BLS. This also applies to the mannequin when considering that chest wall expansion would occur upon reduced gravity exposure, affecting chest compression depth. Other psychological and physiological factors may differ in a simulated study compared to an actual cardiac arrest, such as stress. Furthermore, there are differences in chest wall compliance between humans and mannequins, which do not take into account variations in body anthropometrics, as well as EVA suits. In addition, the sample may not be representative of the commercial space passenger population in terms of demographics.

\section{Conclusion}

In summary, the physiological variables measured showed no significant difference between the 2005 and 2010 BLS

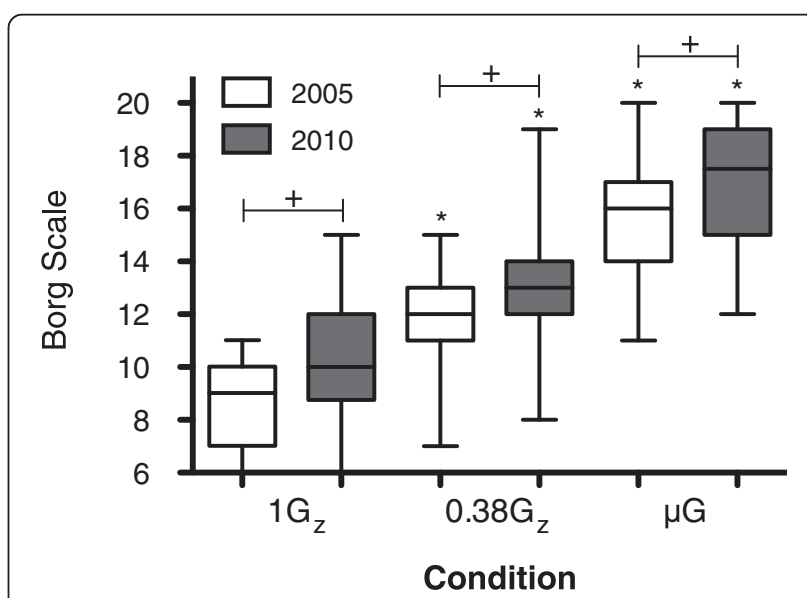

Figure 6 Mean $( \pm S D)$ rate of perceived exertion for four sets of

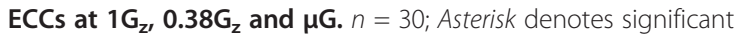
difference to $1 G_{z}$ control, $p<0.05$. Plus sign denotes significant difference between ECC guidelines, $p<0.05$. 
guidelines for all three gravitational conditions studied, although the performance of ECCs during hypogravity and microgravity simulations depicted an increase in physiological cost compared to terrestrial BLS.

This investigation demonstrated that despite ECC $D_{\mathrm{T}}$ and rate being in accordance to the 2005 and 2010 guidelines, accomplishing ECCs in a Martian environment might require a supra-maximal aerobic capacity. Further research into BLS and EVA suits is required to facilitate it on Mars.

Our study also showed that effective ECCs were not attainable for both the ECC guidelines in simulated $\mu \mathrm{G}$ using the ER BLS method. This indicates that future implementation of BLS education using the ER method in simulated $\mu \mathrm{G}$ and upper arm strength training are required to perform effective BLS in space.

Space agencies, commercial space ventures and academic institutions need to collaborate to devise a suitable BLS protocol for hypogravity and microgravity environments, accounting for the difficulty in meeting current terrestrial ECC guidelines in simulated reduced gravity conditions. These findings are even more pertinent with the dawn of commercial spaceflight.

\section{Abbreviations}

ALS: Advanced life support; A-B-C: Airways-breathing-circulation; BLS: Basic life support; BMI: Body mass index; BSD: Body suspension device; CPR: Cardiopulmonary resuscitation; D Recoil: Depth of inadequate recoil; ER: Evetts-Russomano; ECC: External chest compression; EVA: Extravehicular activity; HR: Heart rate; $0.38 \mathrm{G}_{z}$ : Martian hypogravity; $D_{\text {Max: }}$ Maximum depth; $\mu G$ : Microgravity; $V_{E}$ : Minute ventilation; $V O 2$ peak: Peak oxygen consumption; PUCRS: Pontifícia Universidade Catolica do Rio Grande do Sul; $1 \mathrm{G}_{\mathrm{z}}$ : Terrestrial; $D_{\uparrow}$ : True depth.

\section{Competing interests}

The authors declare that they have no competing interests.

\section{Authors' contributions}

TR participated in the design and coordination of the study. JHB participated in the design and coordination of the study, data collection and analysis and helped draft the manuscript. RV participated in the design and coordination of the study, data collection and helped draft the manuscript. RCB participated in data analysis of external chest compressions. AA participated in the data collection and analysis and helped draft the manuscript. LR participated in the data analysis of external chest compressions. RDG recruited volunteers and participated in the data collection and analysis. MKPD recruited volunteers and participated in the data collection and analysis. RRB participated in the design and coordination of the study. All authors read and approved the final manuscript.

Received: 24 July 2012 Accepted: 11 January 2013

Published: 1 April 2013

\section{References}

1. Aubert AE, Beckers F, Verheyden B: Cardiovascular function and basics of physiology in microgravity. Acta Cardiol 2005, 60(2):129-51.

2. Sides MB, Vernikos J, Convertino VA, Stepanek J, Tripp LD, Draeger J, Hargens AR, Kourtidou-Papadeli C, Pavy-LeTraon A: The Bellagio report: cardiovascular risks of spaceflight: implications for the future of space travel. Aviat Space Environ Med 2005, 76(9):877-895.

3. Johnston SL, Marshburn TH, Lindgren K: Predicted incidence of evacuation-level illness/injury during space station operation. In 71st Annual Scientific Meeting of the Aerospace Medical Association: May 2000. Houston, Texas; 2000.
4. Bonin GR: Initiating piloted mars expeditions with medium-lift launch systems. JBIS 2005, 58:302-309.

5. Grenon SM, Xiao X, Hurwitz S, Ramsdell CD, Sheynberg N, Kim C, Williams $\mathrm{GH}$, Cohen RJ: Simulated microgravity induces microvolt T wave alternans. Am J Physiol Heart Circ Physiol 2005, 10(3):363-370.

6. Space tourism market study. http://www.futron.com/spacetourism/default.htm.

7. FAA: 14 CFR Part 417 Launch Safety: Lightning criteria for expendable launch vehicles. Direct Final Rule 2011, 1:33139-33152.

8. Koster RW, Baubin MA, Bossaert LL, Caballero A, Cassan P, Castren M, Granja C, Handley AJ, Monsieurs KG, Perkins GD, Raffay V, Sandroni C: European Resuscitation Council Guidelines for Resuscitation 2010 Section 2. Adult basic life support and use of automated external defibrillators. Resuscitation 2010, 81(10):1277-1292.

9. Hurst V, West S, Austin P, Branson R, Beck G: Comparison of bystander cardiopulmonary resuscitation (BCPR) performance in the absence and presence of timing devices for coordinating delivery of ventilatory breaths and cardiac compressions in a model of adult cardiopulmonary arrest. http://ntrs.nasa.gov/search.jsp?R=20080003861.

10. Dalmarco G, Calder A, Falcão F, de Azevedo DFG, Sarkar S, Evetts S, Moniz S, Russomano T: Evaluation of external cardiac massage performance during hypogravity simulation. In Conf Proc IEEE Eng Med Biol Soc 2006, 1:2904-2907.

11. Evetts SN, Evetts LM, Russomano T, Castro CJ, Ernsting J: Basic life support in microgravity: evaluation of a novel method during parabolic flight. Aviat Space Environ Med 2004, 76:506-510.

12. Borg G: Perceived exertion as an indicator of somatic stress. Scand J Rehabil Med 1970, 2:92-98.

13. Robergs RA, Landwehr $R$ : The surprising history of The HRmax $=220$-age equation. J Exerc Physiol 2002, 5(2):1-10.

14. Rehnberg L, Russomano T, Falcão F, Campos F, Everts SN: Evaluation of a novel basic life support method in simulated microgravity. Aviat Space Environ Med 2011, 82(2):104-110.

15. Kordi M, Cardoso RB, Russomano T: A preliminary comparison between methods of performing external chest compressions during microgravity simulation. Aviat Space Environ Med 2011, 82(12):1161-1163.

16. Day JR, Rossiter HB, Coats EM, Skasick A, Whipp BJ: The maximally attainable $\mathrm{VO}_{2}$ during exercise in humans: the peak vs. maximum issue. J Appl Physiol 2003, 95(5):1901-1907.

17. Moore AD, Lee SMC, Stenger MB, Platts SH: Cardiovascular exercise in the U.S. space program: past, present and future. Acta Astronaut 2010, 66(7-8):974-988.

18. Levine BD, Lane LD, Watenpaugh DE, Gaffney FA, Buckey JC, Blomqvist CG: Maximal exercise performance after adaptation to microgravity. J App/ Physiol 1996, 81(2):686-694.

19. Norcross JR, Lee LR, Clowers KG, Morency RM, Desantis L: DeWitt JK, Jones $J A$, Voss JR, Gernhardt ML: Feasibility of Performing a Suited 10-km Ambulation on the Moon- Final Report of the EVA Walkback Test (EWT). Washington, DC: NASA; 2009.

20. Katuntsev VP, Osipov YY, Filipenkov SN: Biomedical problems of EVA support during manned space flight to Mars. Acta Astronaut 2009, 64(7-8):682-687.

doi:10.1186/2046-7648-2-11

Cite this article as: Russomano et al:: A comparison between the 2010 and 2005 basic life support guidelines during simulated hypogravity and microgravity. Extreme Physiology \& Medicine 2013 2:11. 